\title{
Additive analgesic effect of dexmedetomidine and dezocine administered intrathecally in a mouse pain model
}

\author{
Ya-Qin Huang ${ }^{1,2,3}$, Shao-Hui Guo ${ }^{1}$, Renyu Liu ${ }^{4}$, Sheng-Mei Zhu' ${ }^{1}$, Jian-Liang Sun ${ }^{2}$ \\ and Yong-Xing Yao ${ }^{1,3}$ \\ ${ }^{1}$ Department of Anesthesia, First Affiliated Hospital, Zhejiang University School of Medicine, Hangzhou, P. R. China \\ ${ }^{2}$ Department of Anesthesia, Hangzhou Hospital Affiliated With Nanjing Medical University, Hangzhou First People's Hospital, \\ Hangzhou, P. R. China \\ ${ }^{3}$ Department of Anesthesia, Second Affiliated Hospital of Wenzhou Medical University, Wenzhou, P. R. China \\ ${ }^{4}$ Department of Anesthesiology and Critical Care, Perelman School of Medicine at The University of Pennsylvania, Philadelphia, \\ PA, USA \\ Correspondence to: Yong-Xing Yao, email: yaoyongxing7323@163.com, yaoyongxing@zju.edu.cn \\ Jian-Liang Sun, email: jxmzsj|@163.com \\ Keywords: dexmedetomidine; dezocine; analgesia; acute nociception \\ Received: August 30, $2017 \quad$ Accepted: April 07, $2018 \quad$ Published: May 11, 2018 \\ Copyright: Huang et al. This is an open-access article distributed under the terms of the Creative Commons Attribution License \\ 3.0 (CC BY 3.0), which permits unrestricted use, distribution, and reproduction in any medium, provided the original author and \\ source are credited.
}

\section{ABSTRACT}

Background: It is known that dexmedetomidine can reduce opioid requirements and that there is a synergistic effect when dexmedetomidine and morphine (a full mu opioid receptor agonist) are administered together. However, it was unclear whether a synergistic or additive effect would be observed when dexmedetomidine was co-administered with a partial mu opioid receptor agonist. The present study was designed to elucidate such effects by intrathecally co-administering dexmedetomidine and dezocine, a partial mu receptor agonist, in a mouse pain model.

Methods: C57 mice $(\mathrm{N}=165)$ were randomly divided into 19 groups. The tail flick test was adopted to measure the antinociceptive effects of the tested agents. The mice were divided into saline and drug groups to investigate the dose-dependent analgesic effects. Each drug was administered at fixed doses alone and in combination with one of three doses of a second drug.

Results: Dezocine (0.3125 - $1.25 \mu \mathrm{g})$ and dexmedetomidine $(0.04-1 \mu \mathrm{g})$ both enhanced the tail withdrawal latency in dose-dependent fashions. Dexmedetomidine $(0.04-1 \mu \mathrm{g})$ enhanced the analgesic effect of dezocine. Dezocine (0.3125 - 1.25 $\mathrm{\mu g})$ enhanced the analgesic effect of dexmedetomidine. Compared with the individual drug effects, the combined effects of dezocine $(0.625 \mu \mathrm{g})$ and dexmedetomidine $(0.04 \mu \mathrm{g})$ were more potent 15 - 60 min after injection, but they remained similar to the sum of the effects of the two individual drugs.

Conclusions: Dexmedetomidine and dezocine produce an additive analgesic effect on acute nociception when administered simultaneously.

\section{INTRODUCTION}

Dexmedetomidine is a potent and highly selective agonist of $\alpha_{2}$-adrenergic receptors with analgesic properties against acute inflammatory pain [1], postoperative pain $[2,3]$, and even neuropathic pain that is unresponsive to opioid analgesics [4]. It has also been widely used in clinical settings as an adjuvant for conscious sedation [57]. Previous reports have revealed that dexmedetomidine could reduce opioid requirements and that there is a synergistic effect when dexmedetomidine and morphine (a full mu opioid receptor agonist) are administered together 
$[8,9]$. However, it is unclear whether a synergistic or additive effect can be observed when dexmedetomidine is co-administered with a partial mu receptor agonist. Dezocine, a partial mu opioid receptor agonist and kappa opioid receptor antagonist, produces potent analgesic effects on acute and chronic pain [10-14]. Our recent reports and other studies have suggested that dezocine is at least as effective as morphine and can be used to control moderate to severe pain, such as postoperative pain [ $[15$, 16]. Since it is a partial mu opioid receptor agonist with potential ceiling effects on its responsive receptor, it could be used as a tool to investigate the potential additive or synergistic effects on pain with other medication(s).

In the present study, we investigated the effect of the intrathecal administration of dezocine and dexmedetomidine, two drugs widely used in the perioperative environment, on acute pain conditions via tail flick assays in mice to demonstrate that dezocine and dexmedetomidine have additive or synergistic analgesic effect on nociceptive stimulation.

\section{RESULTS}

\section{Intrathecal dezocine or dexmedetomidine produce dose-dependent antinociception}

Compared with the saline control, dezocine or dexmedetomidine increased the tail withdrawal latency in a dose-dependent fashion, as measured at $15 \mathrm{~min}$ after intrathecal injection, within the dose range of 0.3125 $1.25 \mu \mathrm{g}$ of dezocine $(0 \mu \mathrm{g}, 2.04 \pm 2.04 \% ; 0.3125 \mu \mathrm{g}, 6.94$ $\pm 2.92 \%$; $0.625 \mu \mathrm{g}, 21.53 \pm 4.48 \%$; $1.25 \mu \mathrm{g}, 43.96 \pm 8.15$ $\%$; ANOVA, $F=14.375, P=0.000 ; P=0.015$ vs 0.625 $\mu \mathrm{g}, P=0.009$ vs $1.25 \mu \mathrm{g}, n=7-8$, Figure $1 \mathrm{~A})$ and 0.04 $1 \mu \mathrm{g}$ of dexmedetomidine $(0 \mu \mathrm{g}, 3.7 \pm 1.85 \% ; 0.04 \mu \mathrm{g}$, $21.03 \pm 2.88 \% ; 0.2 \mu \mathrm{g}, 42.92 \pm 9.36 \% ; 1 \mu \mathrm{g}, 95.06 \pm 4.94$ $\%$; ANOVA $F=50.614, P=0.000 ; P=0.035$ vs $0.04 \mu \mathrm{g}$, $P=0.000$ vs $0.2 \mu \mathrm{g}$ and $1 \mu \mathrm{g}, n=9-10$, Figure $1 \mathrm{~B})$.

\section{Dose-dependent analgesic effect of dezocine potentiated by dexmedetomidine and vice versa}

When a fixed dose of $0.625 \mu \mathrm{g}$ dezocine was administered, dexmedetomidine increased the effect of dezocine in a dose-dependent fashion, within a dose range of $0.04-1 \mu \mathrm{g}(0 \mu \mathrm{g}, 20.26 \pm 2.4 \% ; 0.04 \mu \mathrm{g}, 38.03 \pm$ $3.01 \%$; $0.2 \mu \mathrm{g}, 45.92 \pm 6.91 \%$; $1 \mu \mathrm{g}, 92.28 \pm 5.35 \%$; ANOVA, $F=41.336, P=0.000 ; P=0.013$ vs $0.04 \mu \mathrm{g}, P$ $=0.001 v s 0.2 \mu \mathrm{g}, P=0.000 v s 1 \mu \mathrm{g}, n=9$, Figure $2 \mathrm{~A}$ ). When a fixed dose of dexmedetomidine was administered, dezocine increased the effect of dexmedetomidine in a dose-dependent fashion, within a dose range of 0.3125 $1.25 \mu \mathrm{g}(0 \mu \mathrm{g}, 17.72 \pm 4.33 \%$; $0.3125 \mu \mathrm{g}, 22.47 \pm 3.89$ $\%$; $0.625 \mu \mathrm{g}, 35.58 \pm 4.79 \%$; $1.25 \mu \mathrm{g}, 46.69 \pm 8.35 \%$; ANOVA, $F=5.433, P=0.004 ; P=0.032$ vs $0.625 \mu \mathrm{g}, P$ $=0.001 v s 1.25 \mu \mathrm{g}, n=9$, Figure 2B).

\section{Additive effect of dezocine combined with dexmedetomidine in the tail flick assay}

The combined effect of dezocine and dexmedetomidine was more potent at $15 \mathrm{~min}(37.96$ $\pm 4.46 \% ; P=0.043 v s$ dezocine, $23.96 \pm 4.11 \% ; P=$ 0.022 vs dexmedetomidine, $21.96 \pm 5.24 \% ; n=9$, ANOVA, $F=3.550, P=0.045), 30 \mathrm{~min}(47.53 \pm 7.09$ $\% ; P=0.034 v s$ dezocine, $28.17 \pm 3.73 \% ; P=0.0032 v s$ dexmedetomidine, $18.98 \pm 6.81 \% ; n=9$, ANOVA, $F=$ $5.762, P=0.009)$, and $60 \min (33.17 \pm 4.76 \% ; P=0.005$ vs dexmedetomidine, $12.04 \pm 5.66 \% ; n=9$, ANOVA, $F=$ $4.907, P=0.016)$ after administration. However, the effect remained similar to the sum of the two individual effects $(37.96 \pm 4.46 \%$ vs $45.92 \pm 7.17 \%, t=0.942, P=0.360$ at $15 \mathrm{~min} ; 47.53 \pm 7.09 \%$ vs $47.15 \pm 9.61 \%, t=-0.032, P$ $=0.975$ at $30 \mathrm{~min} ; 33.18 \pm 4.76 \%$ vs $34.46 \pm 7.58 \%, t=$ $0.143, P=0.888$ at $60 \mathrm{~min}$ ) (Figure 3 ).

\section{DISCUSSION}

Postoperative pain is the most frequently encountered complaint in postoperative units, and overdoses of opioid analgesics increase the incidence of respiratory depression, hypoxia, and even cardiac arrest. Multi-model analgesic strategies not only reduce the opioid requirement but also reduce the side effects of each medication component.

Pharmacological intervention remains the mainstay for the control of acute pain. In this study, we not only demonstrated that intrathecally administered dezocine or dexmedetomidine alone dose-dependently increased the pain threshold of nociceptive heat stimulation but also found that when added to a fixed dose of dezocine, dexmedetomidine dose-dependently potentiated the analgesic effect of dezocine. When added to a fixed dose of dexmedetomidine, dezocine potentiated the analgesic effect of dexmedetomidine in a dose-dependent fashion. Moreover, we found that the combined use of dezocine with dexmedetomidine produced an effect that was greater than either of their individual effects alone and that was similar to, but not greater than, the sum of their individual effects. Therefore, we deduce that the effect produced by these two drugs, at least at the present doses, is additive rather than synergistic or antagonistic, although we cannot rule out the possibility of other interaction patterns at very low or very high doses.

Once known as a mu opioid receptor antagonist and kappa opioid receptor agonist, dezocine has recently been recognized as a weak mu opioid receptor agonist and kappa opioid receptor antagonist, with an inhibitory effect on the re-uptake of noradrenalin and serotonin [10, $11,17,18]$. Several reports have suggested that dezocine possesses a potent analgesic effect, and thus, it has been used in perioperative pain management $[19,20]$. It has been demonstrated that dezocine's analgesic effect at 
a dose of $10 \mathrm{mg}$ is the same as that of either $50 \mathrm{mg}$ of meperidine or $10 \mathrm{mg}$ of morphine [14]. However, there have been no reports regarding the effect of the interaction between dezocine and the $\alpha_{2}$-adrenergic receptor agonist dexmedetomidine on acute nociception when co-administered intrathecally. Since the use of opioids combined with other medication(s) remains a common practice to control surgical pain, an understanding of the interactive effects of the drugs could be beneficial to patients by enhancing analgesia and reducing side effects. In this study, we discovered that intrathecal co-administration of dexmedetomidine and dezocine

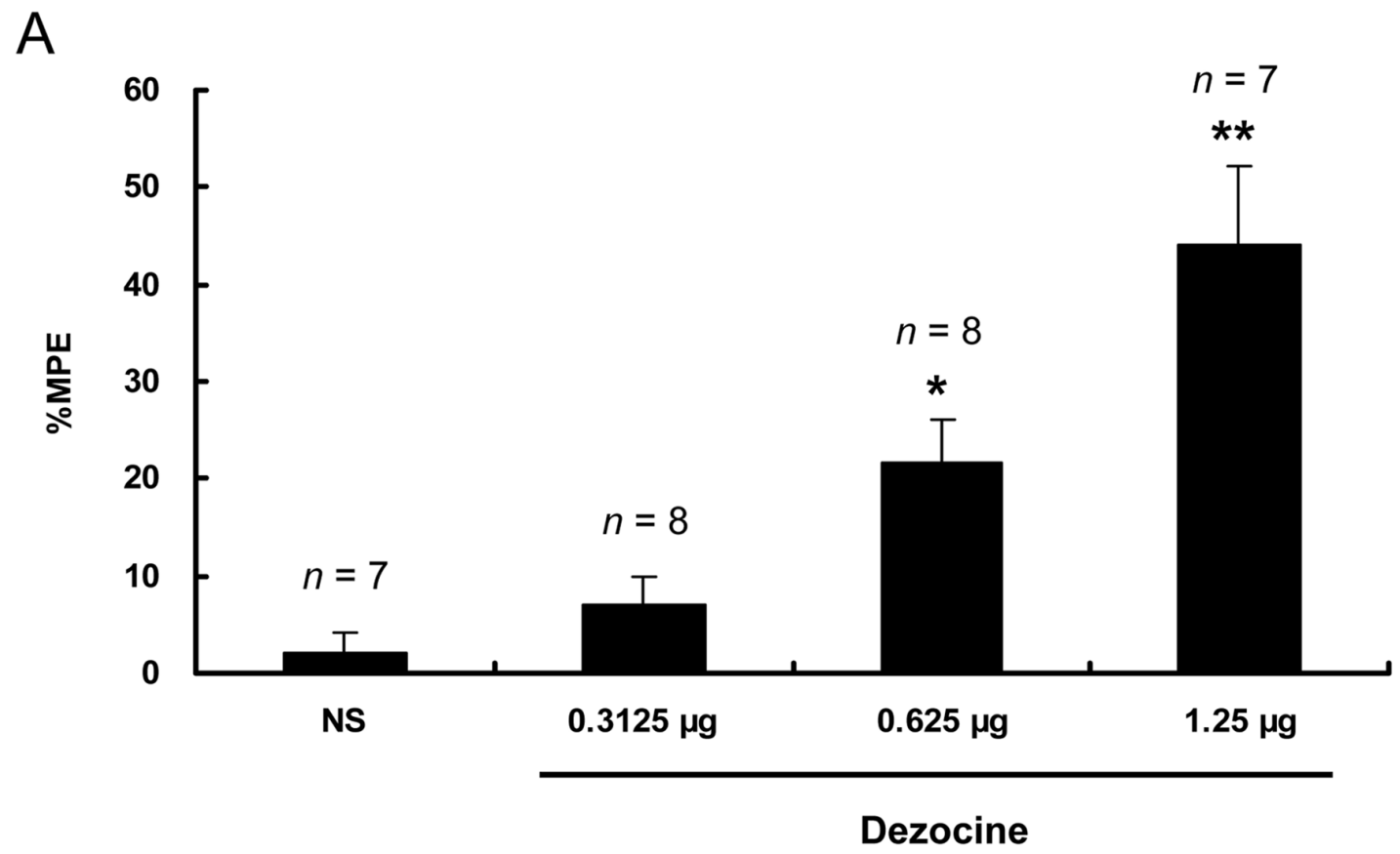

B

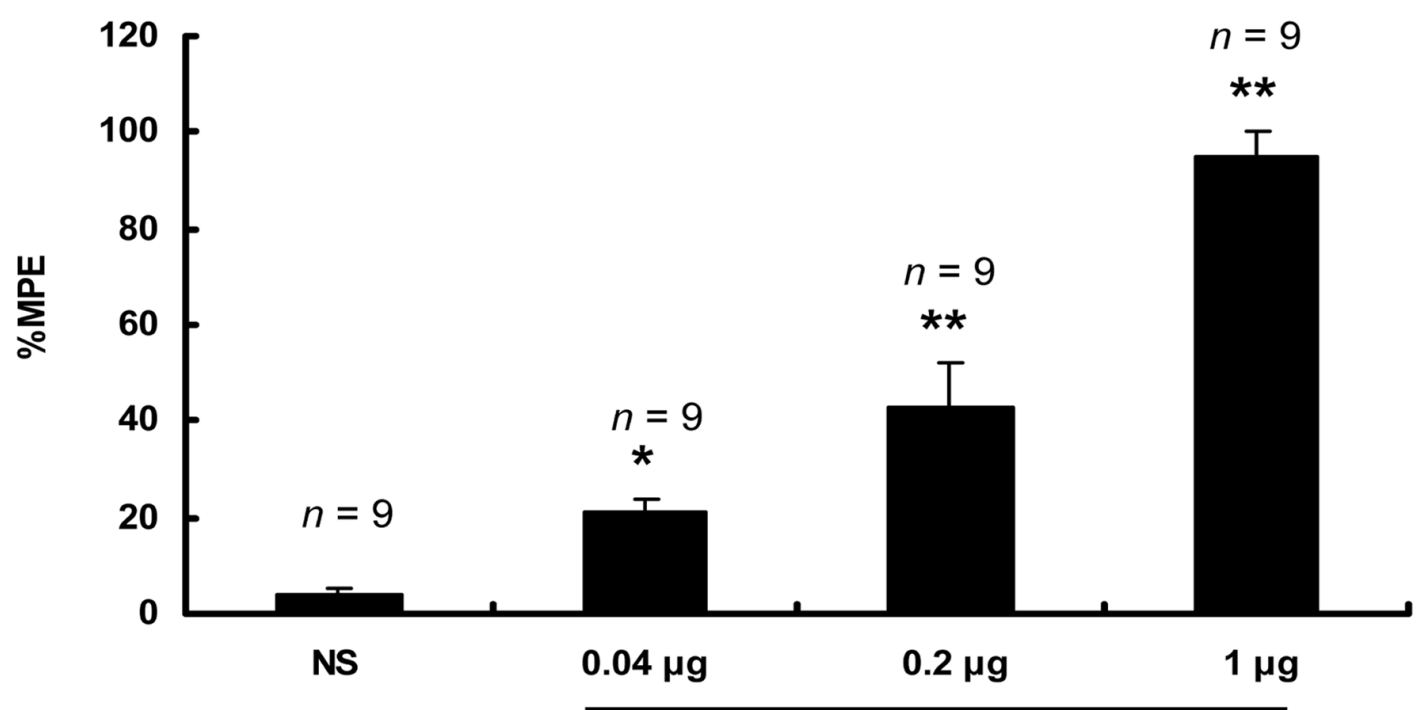

Dexmedetomidine

Figure 1: Intrathecal dezocine or dexmedetomidine produced dose-dependent antinociception in the tail flick test. Within the $0.3125-1.25 \mu \mathrm{g}$ dose range, $15 \mathrm{~min}$ after intrathecal injection, dezocine increased the tail withdrawal latency in a dosedependent fashion (A). Within the $0.04-1 \mu \mathrm{g}$ dose range, dexmedetomidine increased the tail withdrawal latency in a dose-dependent manner (B). ${ }^{*} P<0.05,{ }^{* *} P<0.01$ vs the NS group. Error bars represent the SEM. NS $=$ Normal saline. MPE $=$ Maximum possible effect. 
produced additive analgesic effects, indicating that each drug acts through different receptors in the spinal cord, which is consistent with the known pharmacological effects of both medications.

Dexmedetomidine and dezocine belong two different categories of medications with completely different mechanisms involving different receptors related to pain. Using an additive/synergistic assay, we demonstrated that dezocine combined with dexmedetomidine produced an additive, but not an antagonistic or synergistic, effect on the tail flick test in mice. Thus, it is predicted that dexmedetomidine could reduce the requirements of opioids for analgesic purposes. While both medications produce analgesic effects through receptors in the brain, they also have targets in the spinal cord. This study clearly indicates that they can generate additive effects at the spinal cord level. Although both opioid receptors and alpha-adrenergic receptors belong to the $\mathrm{G}$ protein-coupled receptor family, they play different roles in pain perception. Thus, an additive analgesic effect is somewhat more likely than other interaction patterns. While the analgesic effects of the two drugs are additive, the drugs are not interchangeable. Studies have indicated that there is a synergistic potentiation of antinociceptive effects mediated by morphine and clonidine (an agent similar to dexmedetomidine) [21, 22, 23], although there have been reports showing that adding dexmedetomidine to spinal anesthesia could result in longer motor and
A

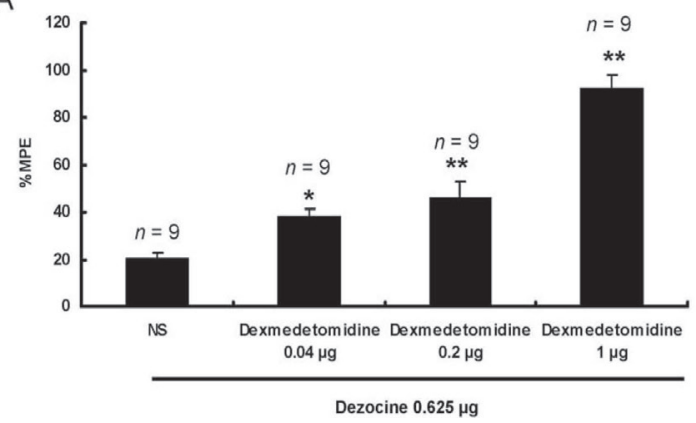

B

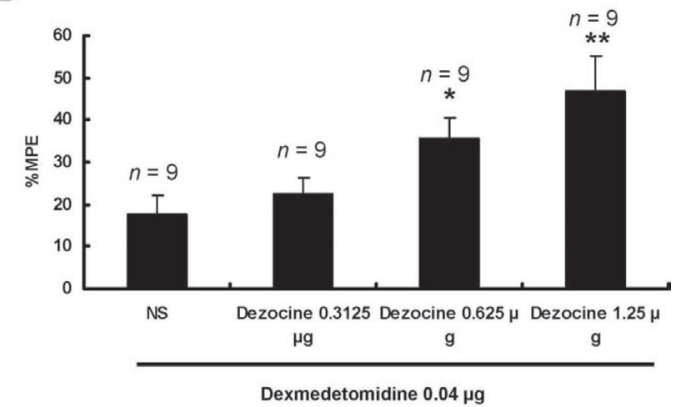

Figure 2: Dose-dependent analgesic effects of dezocine were potentiated by dexmedetomidine and vice versa. At 15 min after intrathecal injection, dexmedetomidine increased the effect of dezocine in a dose-dependent fashion within a dose range of $0.04-1$ $\mu \mathrm{g}$ (A). At $15 \mathrm{~min}$ after intrathecal injection, dezocine increased the effect of dexmedetomidine in a dose-dependent fashion within a dose range of $0.3125-1.25 \mu \mathrm{g}$ (B). ${ }^{*} P<0.05,{ }^{* *} P<0.01 v s$ the NS group. Error bars represent SEM. NS = Normal saline. MPE $=$ Maximum possible effect.

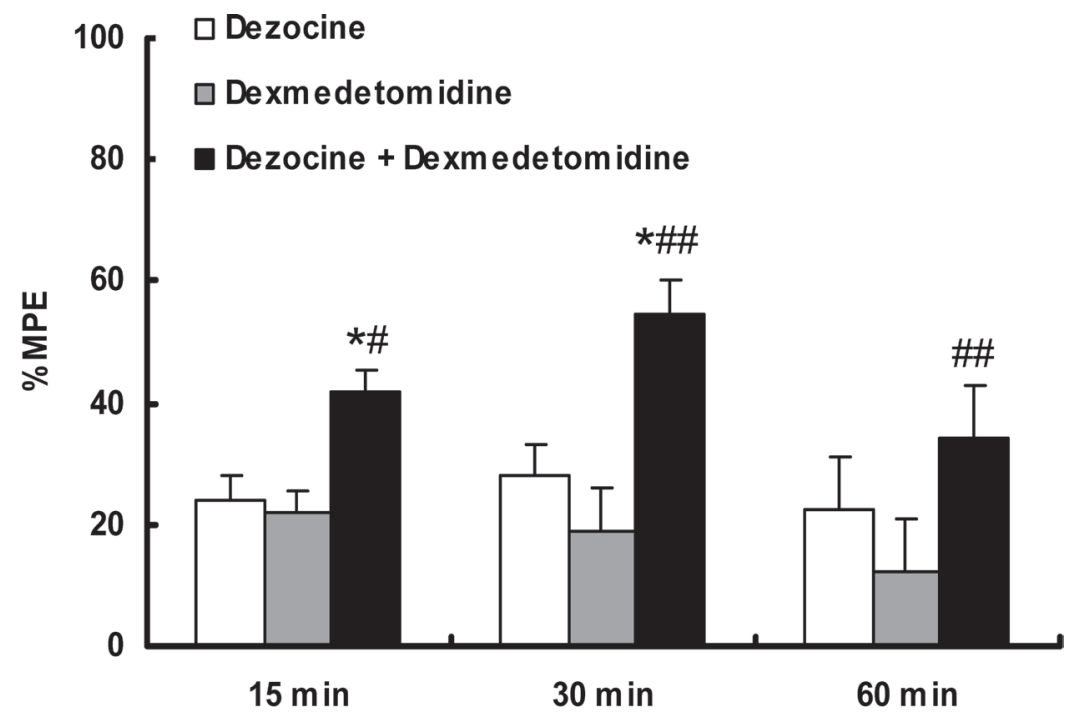

Figure 3: Additive effect of dezocine combined with dexmedetomidine in a tail flick assay. At 30 minutes after injection, the combined effect of dezocine $(0.625 \mu \mathrm{g})$ and dexmedetomidine $(0.04 \mu \mathrm{g})$ was more potent than the effect of dezocine or dexmedetomidine alone. However, this effect remained similar to the sum of the two individual effects. ${ }^{*} P<0.05$ vs dezocine, ${ }^{\#} P<0.05$, ${ }^{\#} P<0.01 v s$ dexmedetomidine, $n=9$. Error bars represent the SEM. NS = Normal saline. MPE = Maximum possible effect. 
sensory blocks than the addition of narcotics [24]. The major difference between morphine and dezocine as pain medication is that morphine is a pan-agonist that activates all the opioid receptors, including kappa opioid receptors, while dezocine is a partial mu receptor agonist and kappa opioid receptor antagonist. It is unclear whether dexmedetomidine interacts with opioid receptors in the spinal cord. If dexmedetomidine interacts with opioid receptors, especially kappa opioid receptors, as a full agonist, it would be potential mechanism to explain why morphine and dexmedetomidine have synergistic effects. Molecular target studies need to be performed to investigate that possibility. In clinical practice, additive effects may be more desirable than synergistic effects, since they are more predictable, making it easier to avoid undesirable side effects. However, the present results were obtained in animal models, and clinical studies are necessary to further elucidate the interactions and the potential side effects, such as over-sedation or respiratory depression, of this combination of drugs.

\section{MATERIALS AND METHODS}

Animal use and care, as well as the experimental protocols, were reviewed and approved by the Ethics Committee of the First Affiliated Hospital, Zhejiang University School of Medicine. These studies were also consistent with the ethical guidelines for the investigation of experimental pain in animals [25]. All possible efforts were made to minimize the number of animals used and their suffering.

\section{Animals}

Male adult C57 mice ( $N=165$; body weight $\sim 25$ g) were obtained from the Animal Center of the Chinese Academy of Sciences (Shanghai, P. R. China). The animals were housed with 10 mice per cage with water and food available ad libitum and were maintained under a 12-h light/12-h dark cycle with the lights turned on at 08:00 AM.

\section{Tail flick test}

The tail withdrawal latency was assessed as previously reported [26]. Briefly, the mice were gently restrained along the edge of a table, and their tails were immersed in water, which was maintained at $52^{\circ} \mathrm{C}$ using hot water baths. The latency to tail withdrawal was measured using a hand-held stopwatch. One hour after the baseline was determined, medications $(5 \mu \mathrm{l}$ in total volume) were intrathecally administered via direct lumbar puncture, according to the procedure described by Hylden and Wilcox in 1980 [27]. Briefly, a modified $25 \mu \mathrm{l}$ microsyringe was inserted between the L5 and L6 vertebrae of conscious mice. A sudden flick of the tail indicated the success of the puncture into the subarachnoid space. The drug solution or vehicle was injected over a period of $30 \mathrm{~s}$. The tail withdrawal latency was re-determined at 15,30 or 60 min after drug administration. To avoid tissue damage, a maximum score $(100 \%)$ was assigned to the animals that did not respond within $10 \mathrm{~s}$. Antinociception was calculated by the following formula: \% maximum possible effect $(\mathrm{MPE})=100 \times($ test latency - baseline latency $) /(10$ - baseline latency) [28].

\section{Medications}

Dezocine was obtained from the Yangtze River Pharmaceutical Group Co., Ltd., Jiangsu, P. R. China. Dexmedetomidine was obtained from the Jiangsu Hengrui Medicine Co., Ltd., Jiangsu, P. R. China.

The animals were randomly allocated to each group. The sample size was estimated according to previous reports [28, 29]. Both dezocine and dexmedetomidine were administered intrathecally. To determine the dosedependent effects on antinociception, the mice were divided into saline control, dezocine $(0.3125,0.625$, or $1.25 \mu \mathrm{g})$, or dexmedetomidine $(0.04,0.2$, or $1 \mu \mathrm{g}$ ) groups. To investigate the interaction between dezocine and dexmedetomidine, dezocine was administered at a fixed dose of $0.625 \mu \mathrm{g}$ and combined with various doses of dexmedetomidine $(0.04,0.2$, or $1 \mu \mathrm{g})$ in one set of experiments. Dexmedetomidine was administered at a fixed dose of $0.04 \mu \mathrm{g}$ and combined with various doses of dezocine $(0.3125,0.625$, or $1.25 \mu \mathrm{g})$ in another set of experiments. The additive/synergistic effect was deduced via comparison of the combined effect with the sum of the two individual effects, according to previous reports $[16,29-31]$.

\section{Statistical analysis}

The data are expressed as the mean \pm standard error of the mean (SEM) and were analyzed with oneway analysis of variance (ANOVA), followed by the least significant difference test for multiple comparisons (LSD). To test additivity in the one-dose experiments, the mean values and standard variations of the combined single-drug groups were compared with the values of the respective combination group using independent $t$-tests. A $P$-value $<0.05$ was considered statistically significant.

\section{CONCLUSIONS}

In conclusion, dezocine and dexmedetomidine produce dose-dependent antinociceptive effects in mice. When simultaneously administered intrathecally, dezocine and dexmedetomidine produce an additive effect on acute nociception. These results suggest that dexmedetomidine can be used to enhance the analgesic effects of dezocine 
and/or to reduce the requirement for dezocine for acute pain management in the perioperative period.

\section{Author contributions}

Yao YX and Sun JL contributed to the study design. Yao YX and Huang YQ conducted the study. Guo SH and Zhu SM collected and analyzed the data. Yao YX drafted the manuscript. Liu R made critical revisions of the manuscript. All authors read and approved the final manuscript.

\section{CONFLICTS OF INTEREST} interests.

The authors declare that they have no competing

\section{FUNDING}

This work was supported by the National Natural Science Foundation of China (NSFC) (grants No. 30972846 and 81471127) and the Talent Project of the Zhejiang Provincial Department of Health (grants No. 2012RCB018 and 2013RCA016).

\section{REFERENCES}

1. Xu B, Zhang WS, Yang JL, Lû N, Deng XM, Xu H, Zhang YQ. Evidence for suppression of spinal glial activation by dexmedetomidine in a rat model of monoarthritis. Clin Exp Pharmacol Physiol. 2010; 37: e158-66.

2. Shimode N, Fukuoka T, Tanimoto M, Tashiro C, Tokunaga A, Noguchi K. The effects of dexmedetomidine and halothane on Fos expression in the spinal dorsal horn using a rat postoperative pain model. Neurosci Lett. 2003; 343: 45-8.

3. Hwang W, Lee J, Park J, Joo J. Dexmedetomidine versus remifentanil in postoperative pain control after spinal surgery: a randomized controlled study. BMC Anesthesiol. 2015; 15: 21.

4. Liu L, Ji F, Liang J, He H, Fu Y, Cao M. Inhibition by dexmedetomidine of the activation of spinal dorsal horn glias and the intracellular ERK signaling pathway induced by nerve injury. Brain Res. 2012; 1427: 1-9.

5. Schnabel A, Meyer-Frießem CH, Reichl SU, Zahn PK, Pogatzki-Zahn EM. Is intraoperative dexmedetomidine a new option for postoperative pain treatment? A metaanalysis of randomized controlled trials. Pain. 2013;154: 1140-9.

6. Fan Q, Hu C, Ye M, Shen X. Dexmedetomidine for tracheal extubation in deeply anesthetized adult patients after otologic surgery: a comparison with remifentanil. BMC Anesthesiol. 2015;15:106.

7. Su X, Meng ZT, Wu XH, Cui F, Li HL, Wang DX, Zhu X, Zhu SN, Maze M, Ma D. Dexmedetomidine for prevention of delirium in elderly patients after non-cardiac surgery: a randomised, double-blind, placebo-controlled trial. Lancet. 2016; 388:1893-902.

8. Kabalak A, Ekmekçioğlu E, Ceylan A, Kahveci K. The synergistic antinociceptive interactions of morphine and dexmedetomidine in rats with nerve-ligation injury. Hippokratia. 2013; 17: 326-31.

9. Bae HB. Dexmedetomidine: an attractive adjunct to anesthesia. Korean J Anesthesiol. 2017; 70:375-6.

10. Gharagozlou P, Demirci H, Clark DJ, Lameh J. Activity of opioid ligands in cells expressing cloned mu opioid receptors. BMC Pharmacology. 2003; 3: 1.

11. Gharagozlou P, Hashemi E, DeLorey TM, Clark JD, Lameh J. Pharmacological profiles of opioid ligands at kappa opioid receptors. BMC Pharmacology. 2006; 6: 3.

12. Gal TJ, DiFazio CA. Ventilatory and analgesic effects of dezocine in humans. Anesthesiology. 1984; 61: 716-22.

13. Wu FX, Pan RR, Yu WF, Liu R. The Anti-Nociception Effect of Dezocine in a Rat Neuropathic Pain Model. Transl Perioper Pain Med. 2014; 1: 5-8.

14. Wu FX, Babazada H, Gao H, Huang XP, Xi CH, Chen CH, Xi J, Yu WF, Liu R. Dezocine Alleviates Morphine-Induced Dependence in Rats. Anesth Analg. 2018 Apr 5. https://doi. org/10.1213/ANE.0000000000003365. [Epub ahead of print]

15. O'Brien JJ, Benfield P. Dezocine. A preliminary review of its pharmacodynamic and pharmacokinetic properties, and therapeutic efficacy. Drugs. 1989; 38: 226-48.

16. Li NN, Huang YQ, Huang LE, Guo SH, Shen MR, Guo CL, Zhu SM, Yao YX. Dezocine Antagonizes Morphine Analgesia upon Simultaneous Administration in Rodent Models of Acute Nociception. Pain Physician. 2017; 20: E401-9.

17. Liu R, Huang XP, Yeliseev A, Xi J, Roth BL. Novel molecular targets of dezocine and their clinical implications. Anesthesiology. 2014; 120: 714-23.

18. Wang YX, Mao XF, Li TF, Gong N, Zhang MZ. Dezocine exhibits antihypersensitivity activities in neuropathy through spinal $\mu$-opioid receptor activation and norepinephrine reuptake inhibition. Sci Rep. 2017; 7: 43137.

19. Cohen RI, Edwards WT, Kezer EA, Ferrari DA, Liland AE, Smith ER. Serial intravenous doses of dezocine, morphine, and nalbuphine in the management of postoperative pain for outpatients. Anesth Analg. 1993; 77: 533-9.

20. Zhu Y, Jing G, Yuan W. Preoperative administration of intramuscular dezocine reduces postoperative pain for laparoscopic cholecystectomy. J Biomed Res. 2011; 25: 356-61.

21. Wilcox GL, Carlsson KH, Jochim A, Jurna I. Mutual potentiation of antinociceptive effects of morphine and clonidine on motor and sensory responses in rat spinal cord. Brain Res. 1987; 405: 84-93.

22. Sullivan AF, Dashwood MR, Dickenson AH. Alpha 2-adrenoceptor modulation of nociception in rat spinal 
cord: location, effects and interactions with morphine.Eur J Pharmacol. 1987; 138: 169-77.

23. Ossipov MH, Suarez LJ, Spaulding TC. Antinociceptive interactions between alpha 2-adrenergic and opiate agonists at the spinal level in rodents. Anesth Analg. 1989; 68: 194-200.

24. Gupta R, Verma R, Bogra J, Kohli M, Raman R, Kushwaha JK. A Comparative study of intrathecal dexmedetomidine and fentanyl as adjuvants to Bupivacaine. J Anaesthesiol Clin Pharmacol. 2011; 27: 339-43.

25. Zimmermann M. Ethical guidelines for investigations of experimental pain in conscious animals. Pain. 1983; 16: 109-10.

26. Hopkins E, Rossi G, Kest B. Sex differences in systemic morphine analgesic tolerance following intrathecal morphine injections. Brain Res. 2004; 1014: 244-6.

27. Hylden JL, Wilcox GL. Intrathecal morphine in mice: a new technique. Eur J Pharmacol. 1980; 67: 313-6.
28. Zhao ZQ, Gao YJ, Sun YG, Zhao CS, Gereau RW 4th, Chen ZF. Central serotonergic neurons are differentially required for opioid analgesia but not for morphine tolerance or morphine reward. Proc Natl Acad Sci U S A. 2007;104: 14519-24.

29. Plummer JL, Cmielewski PL, Gourlay GK, Owen $\mathrm{H}$, Cousins MJ. Antinociceptive and motor effects of intrathecal morphine combined with intrathecal clonidine, noradrenaline, carbachol or midazolam in rats. Pain. 1992; 49:145-52.

30. Idänpään-Heikkilä JJ, Kalso EA, Seppälä T. Antinociceptive actions of dexmedetomidine and the kappa-opioid agonist $\mathrm{U}-50,488 \mathrm{H}$ against noxious thermal, mechanical and inflammatory stimuli. J Pharmacol Exp Ther. 1994; 271: 1306-13.

31. Morgan D, Cook CD, Smith MA, Picker M. An examination of the interactions between the antinociceptive effects of morphine and various mu-opioids: the role of intrinsic efficacy and stimulus intensity. Anesth Analg. 1999; 88: 407-13. 